\title{
Study of flaring quasars using optical/gamma-ray correlations
}

\section{Richard J. Britto*}

Department of Physics, University of Johannesburg, Auckland Park 2006, South Africa

E-mail: rbritto@uj.ac.za, dr.richard.britto@gmail.com

Flat spectrum radio quasars (FSRQs) are active galactic nuclei where one of the plasma jets is directed towards the Earth's direction. They are surrounded by a thick shell of gas clouds called the broad-line region (BLR) - in which gamma rays are expected to be absorbed through the photon-photon pair production between UV photons from the BLR and gamma rays from the jets. These sources are found at high redshifts and have displayed light curves with highly variable patterns, with fluxes that can increase to several times the quiescent state of the object in a few minutes. Many FSRQs are very bright in gamma-rays (at the $\mathrm{MeV}$ and $\mathrm{GeV}$ energy ranges). During flaring periods, correlation between radio, optical and gamma-ray fluxes are regularly observed, that show the dependency between synchrotron emission (optical) and inverse Compton emission (gamma rays). Also, spectra from infrared to ultraviolet bands can unveil features of the BLR used to model the absorption of gamma rays above $\sim 10 \mathrm{GeV}$. In this paper a list of FSRQs is presented, that can be observed by the Southern African Large Telescope (SALT), as well as examples of correlation studies and spectral modeling that can be achieved by a quasisimultaneous optical / gamma-ray monitoring.

SALT Science Conference 2015 -SSC2015-

1-5 June, 2015

Stellenbosch Institute of Advanced Study, South Africa

\footnotetext{
*Speaker.
} 


\section{Introduction}

Active Galactic Nuclei (AGNs) are powerful compact objects at the centre of mainly elliptical galaxies. They are understood as supermassive $\left(\sim 10^{6}-10^{9}\right.$ solar mass $)$ black holes surrounded by an accretion disk and other material up to a few parsecs. Radio loud AGNs exhibit a pair of twin jets of ultra-relativistic plasma, and radiation spread over the whole electromagnetic spectrum (Figure 1). These jets are perpendicular to the accretion disk. The most energetic AGNs are called quasars (acronym for quasi-stellar radio sources), and quasars whose jets are close to the direction of the Earth are called blazars (e.g., Urry \& Padovani [24] and Finke [14] for reviews). Blazars are particularly bright in gamma rays, and exhibit a dramatic flux variability pattern during flaring activity, sometimes as short as sub-hour time scales. A sub-class of blazar, called flat spectrum radio quasars (FSRQs), are surrounded by a thick shell of gas clouds - called the broad-line region (BLR) - in which gamma rays are expected to be absorbed above $\sim 10 \mathrm{GeV}$ through the photon-photon pair production between UV photons from the BLR and gamma rays from the jets $[19,21,7]$. FSRQs are usually more luminous than BL Lacs (the other sub-class of blazars).

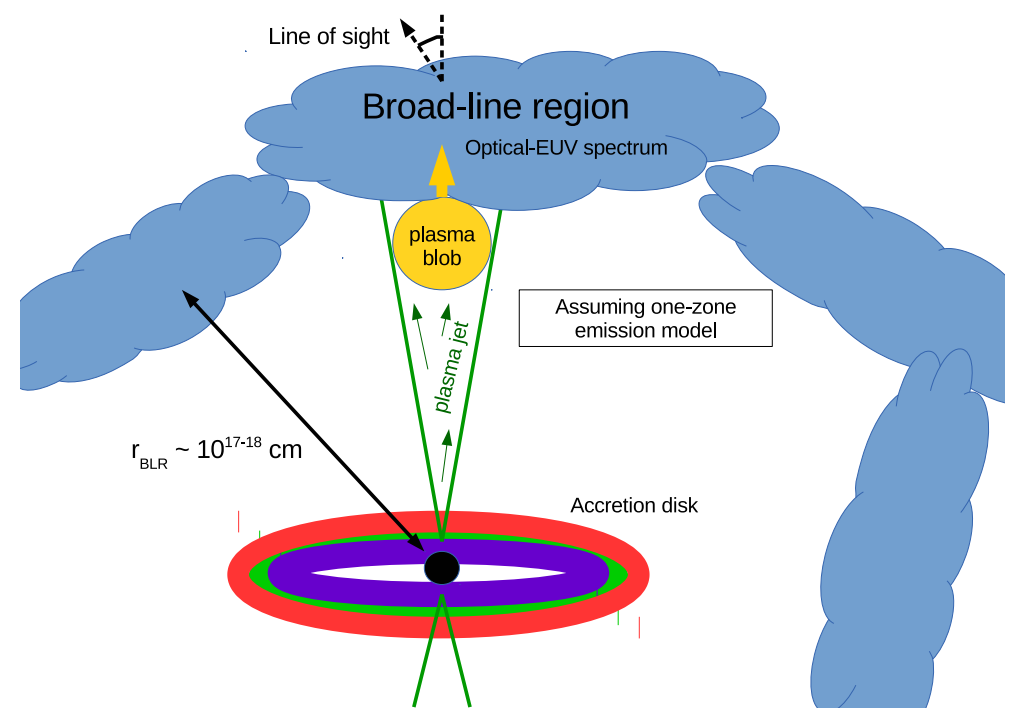

Figure 1: Cartoon of a flaring FSRQ, with the gamma-ray emitting region (plasma blob) below the BLR in this example. This figure was drawn in the context of the unified model of AGN, and with the assumption that the blob size equals the jet cross section. Colors of the accretion disk represent the UV to IR components, from the inner to outer disk.

Modeling the spectral energy distributions of FSRQs can be quite challenging, due to the presence of the BLR, in which, during flaring episodes, gamma rays may be absorbed, depending on the location of the gamma-ray emitting plasma blob. This blob may be located within or beyond the BLR. The determination of the location of the blob and of the gamma-ray absorption level are tools to understand mechanisms of radiation production in AGNs. A calculation of the gamma-ray absorption by the BLR radiation field should rely on a reasonable model of the BLR spectra, such as the composite spectra obtained by Telfer et al. [23].

The Fermi Large Area Telescope (Fermi-LAT) is a gamma-ray pair conversion telescope in 
orbit around the Earth since 2008, monitoring the whole sky in survey mode for most of its operating mode [6]. It is sensitive to photons in the $20 \mathrm{MeV}-300 \mathrm{GeV}$ energy range, and has reported the detection of 3033 sources above $\sim 4 \sigma$ (test statistic $>25$ ) in the first 4 years of mission (3FGL, [1]). The whole AGN sample from 3FGL, detected on the whole sky, consists of 1773 sources, including 491 FSRQs. Acero et al. (3LAC catalog paper: [3]) reported the study of the AGN sample lying at Galactic latitude $b \geq 10 \mathrm{deg}$. The telescope's capability to see the whole sky every three hours makes Fermi-LAT a first choice instrument for the time-domain study of flaring episodes.

\section{Optical / gamma-ray correlations in light curves}
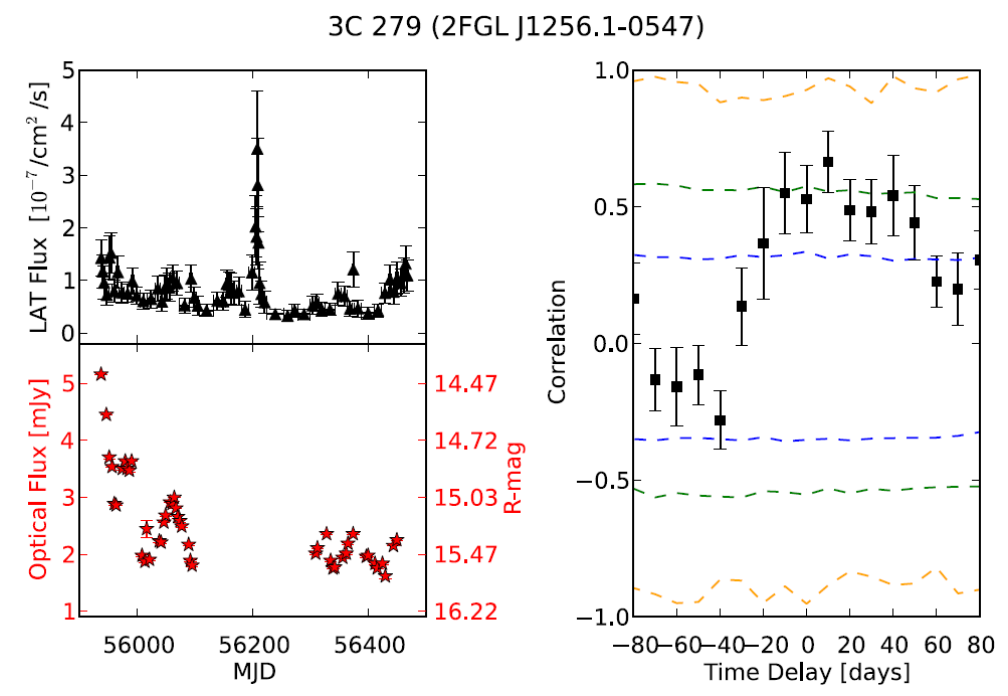

Figure 2: Fermi-LAT light-curve (top left), KAIT light-curve (bottom left) and DCF (right) for FSRQ 3C 279. Blue, green, and orange dashed lines on the DCF plot represent $68 \%, 90 \%$, and $99 \%$ significance levels, respectively. This source has a DCF peak (92.3\% significance) at $\tau=19.7 \pm 3.4$ days. Unfortunately, optical coverage missed a very large gamma-ray flare at MJD $\approx 56200$, so the correlation here is dominated by smaller-scale variability. Figure 4 from Cohen et al. [12].

It is quite common to obtain Fermi-LAT light-curves in sub-day binning during prominent flares of bright FSRQs (e.g., [2, 5, 20, 16, 8]). When regular UV to IR monitoring of these flaring episodes is performed, we can study quasi-simultaneous light-curves and undertake correlation studies between optical and gamma rays, that provides constraints on mechanisms of blazar radiation production and on the location of the emission regions.

An interesting study was undertaken by Cohen et al. [12] for the flares of 40 bright Fermi-LAT blazars, using data from LAT and the $0.76 \mathrm{~m}$ Katzman Automatic Imaging Telescope (KAIT). Time lags between optical and gamma rays were calculated using the Discrete Correlation Function (DCF) [13]. In Figure 2 is illustrated the correlation study that these authors performed for 3C 279. For this flare, the time lag $\tau=19.7 \pm 3.4$ days of gamma-ray leading the optical is found with a DFC peak of $92.3 \%$ significance, although optical coverage is missing during the brightest gammaray flare. Positive values of $\tau$ were found to be a general trend for FSRQs, supporting the leptonic model of blazar emission with external Compton scattering. 


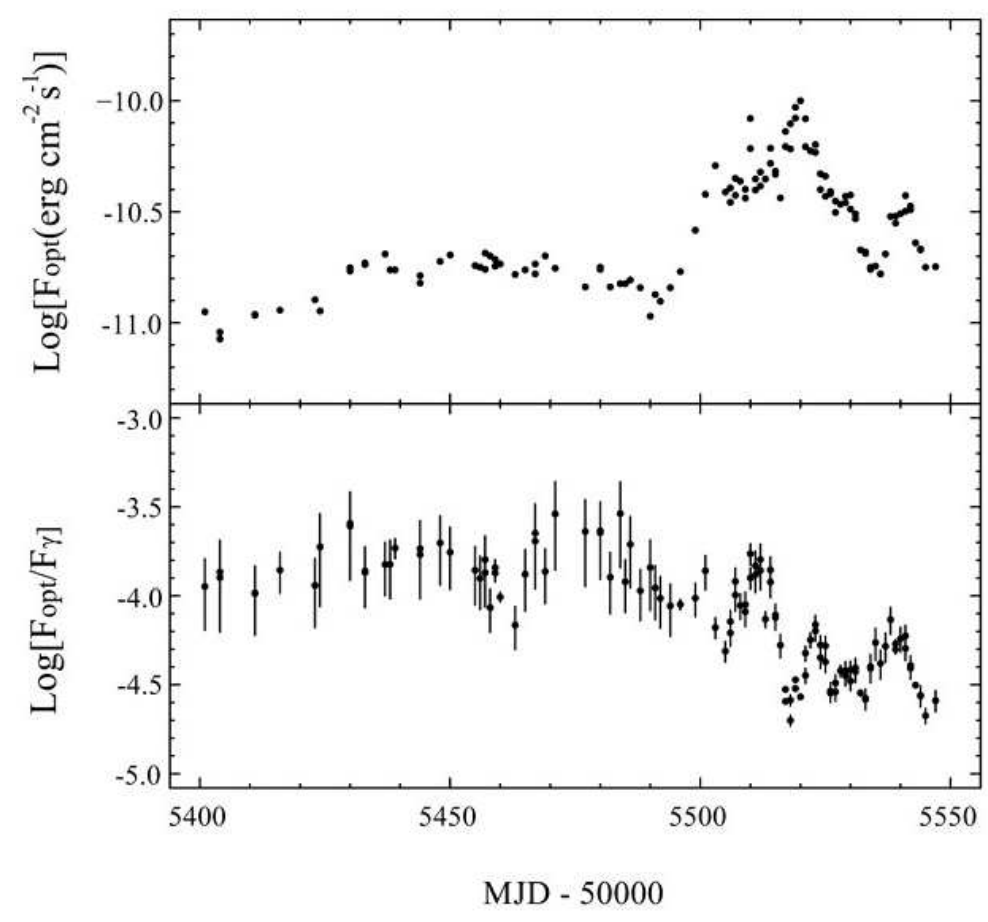

Figure 3: Light-curves of 3C 454.3 in the MDJ 55400-55550 time period. Upper panel: R-band light-curve. Lower panel: Ratio $F_{o p t} / F_{\gamma}$. Figure 5 from Tachibana et al. [22].

Tachibana et al. [22] studied two major flares of 3C 454.3. Part of their study is based on the flux ratio $F_{\text {opt }} / F_{\gamma}$ (Figure 3), used to investigate the role of the Doppler factor $\delta$ in this flux ratio variability.

Also, a similar approach of light-curve correlation studies using DCFs was carried out by Fuhrmann et al. [15], by using centimeter to sub-millimeter radio data, along with Fermi-LAT data.

\section{Flux variability in optical spectra}

León-Tavares et al. [17] studied the flux-like variability of the $\mathrm{Mg}$ II $\lambda 2800$ emission line of FSRQ 3C 454.3 by acquiring optical spectra as part of the Ground-based Observational Support of the Fermi Gamma-Ray Space Telescope at the University of Arizona monitoring programme. In Figure 4 is presented the rest-frame optical spectra of 3C 454.3 in three intensity states, including the giant flare of November 2010. Knowing the luminosity of the BLR during the appropriate flaring episodes can improve the modeling of this radiation field in order to make more accurate predictions of $\mathrm{GeV}$ to $\mathrm{TeV}$ absorption, compared to a generic model built on a composite spectrum of radio-loud quasars. 

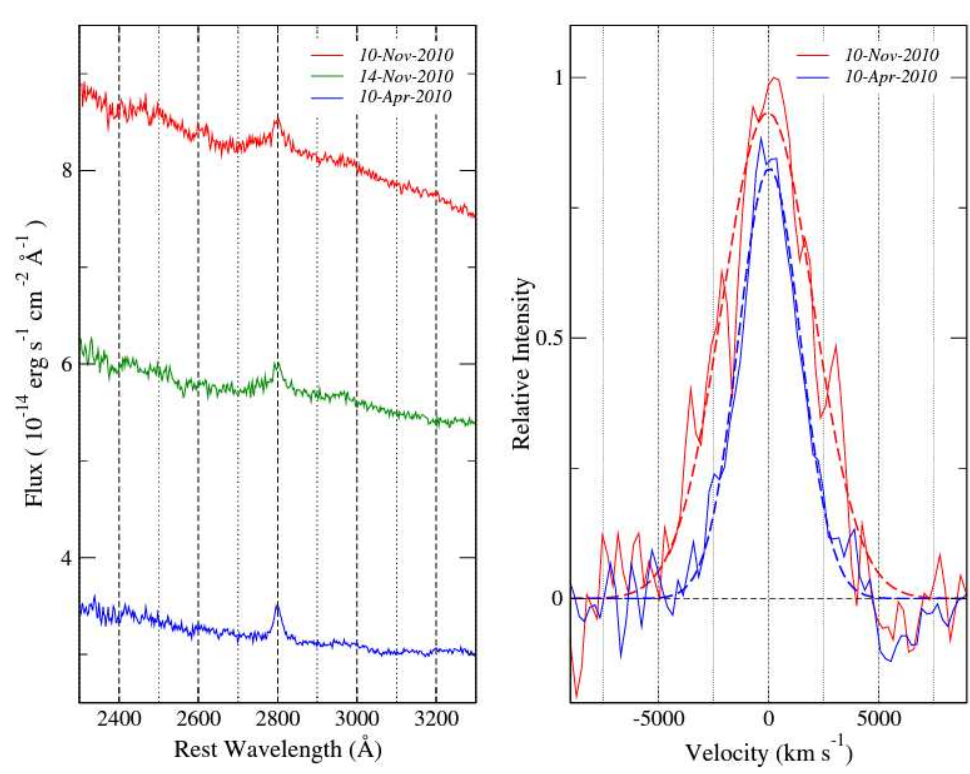

Figure 4: Left: rest-frame optical spectra of 3C 454.3 in three intensity states. As can be seen from the top spectrum, the $\mathrm{Mg}$ II $\lambda 2800$ emission line is detectable, despite the high levels of optical continuum emission observed. Right: comparison of the variations seen in the $\mathrm{Mg}$ II $\lambda 2800$ profiles (after continuum and Fe II subtraction) from 2010 April to November. The observed spectra are shown in solid line and a fitted Gaussian to the profile are shown in dashed lines. Observing times are color coded as shown in the legend. Figure 1 of León-Tavares et al. [17].

\section{Perspectives with SALT}

Small telescopes of the South African Astronomical Observatory (SAAO) can be used to acquire photometric data and produce optical light-curves with several filters. However, optical spectra can be obtained with SALT (needed to allow rapid targets of opportunity (ToO) followup).

The Southern African Large Telescope (SALT) is a 11-m wide telescope, located at the SAAO (latitude: $32^{\circ} 22^{\prime} 33.62^{\prime \prime} \mathrm{S}$; longitude: $20^{\circ} 48^{\prime} 38.44^{\prime \prime} \mathrm{E}$ ) [9]. SALT is funded by a consortium of international partners from seven countries: South Africa, the United States, Germany, Poland, India, the United Kingdom and New Zealand. The telescope has been fully operational since September 2011, and has already been the source of several discoveries due to this new potential of astronomical observations in the Southern hemisphere. The Robert Stobie Spectrometer (RSS) is SALT's powerful instrument for high quality spectrometry, sensitive to the 320-900 nm wavelength range. This instrument can achieve resolutions up to $R \sim 5000$ with 1 arcsec slits, and $R \sim 9000$ with 0.6 arcsec slits [11].

To benefit from SALT's capability in the optical, I selected a sample of FSRQ candidates which can be observed by SALT-RSS, i.e., within the declination range -75 to $+10 \mathrm{deg}$. In order to establish a list of FSRQs to be used for the proposed optical/gamma-ray correlation study, I selected sources detected by Fermi-LAT. Also, for possible TeV counterpart, or at least studies at $\mathrm{GeV}$ energies with a reasonable statistics, I searched for sources with a hard spectrum, using sources from the Second Catalog of Hard Fermi-LAT Sources (2FHL, [4]), which includes sources 


\begin{tabular}{|c|c|c|c|}
\hline Source & declination & redshift & TeV detection \\
\hline PKS 1510-089 & $-08.9 \mathrm{deg}$ & 0.360 & yes \\
3C 279 & $-05.8 \mathrm{deg}$ & 0.536 & yes \\
PKS 0454-234 & $-23.4 \mathrm{deg}$ & 1.003 & no \\
PKS 1424-418 & $-41.8 \mathrm{deg}$ & 1.522 & no \\
\hline NVSS J141922-083830 & $-08.4 \mathrm{deg}$ & $0.903 ?$ & no \\
\hline
\end{tabular}

Table 1: Our proposed ToOs for SALT and optical/gamma-ray correlation studies.

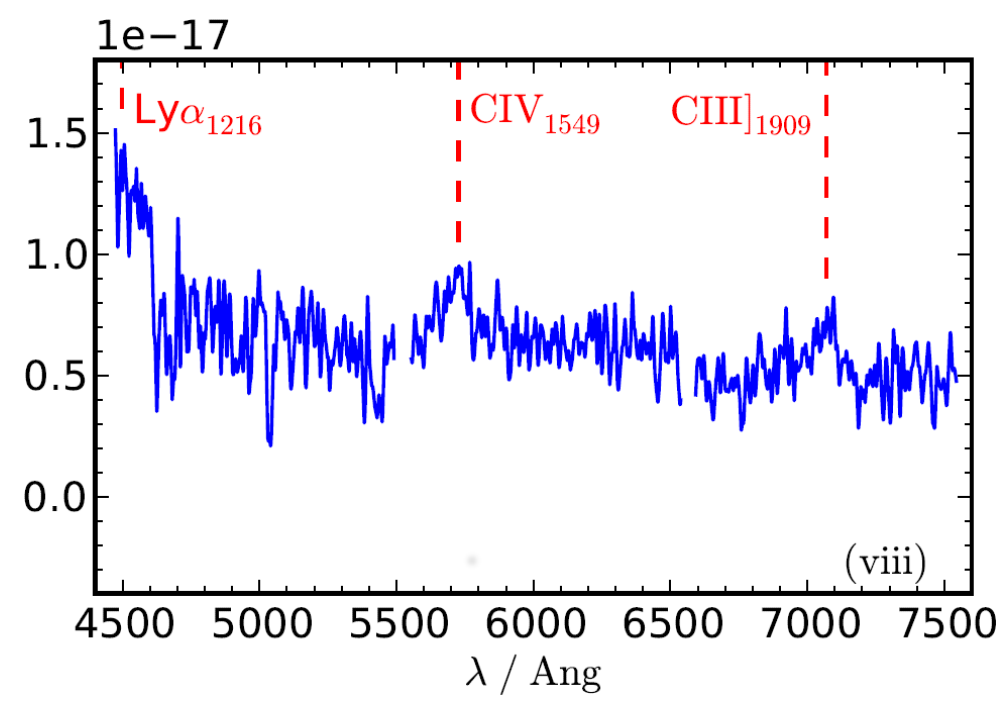

Figure 5: SALT spectrum for a quasar at $z=2.7\left(\mathrm{RA}=02 \mathrm{~h} 27 \mathrm{~m} 09.03 \mathrm{~s}\right.$, Dec $\left.=-04^{\circ} 55^{\prime} 10.1^{\prime \prime}\right)$. On the y-axis, flux $F_{\lambda}$ is given in [erg cm $\mathrm{cm}^{-2} \mathrm{~s}^{-1} \AA^{-1}$. Sub-set of Figure D1 of White et al. [25].

detected above $50 \mathrm{GeV}$ in the first six years of the Fermi mission (2008-2014). Hard sources (bright at high energies) would allow us to investigate gamma-ray absorption in the BLR. Also, an additional source, NVSS J141922-083830 (2FGL J1419.4-0835), which is a candidate FSRQ, has been added to the list. Although this source is not particularly bright, nor is it a 2FHL object, it was observed in Feb 2015 as MASTER-SAAO optical transient [18], and a spectrum with SALTRSS has been obtained [10]. This gives a perspective for a more complete characterization of this source, along with the potential for optical/gamma-ray correlation studies. In Table 1 are listed the proposed ToOs for combined spectral and variability studies, using SAAO/SALT and Fermi-LAT data sets.

As introduced in Section 3, monitoring flux variability of spectral lines is an interesting way to probe features and spatial spread of the BLR. If we study high redshift blazars $(z \gtrsim 1.7)$, the Ly $\alpha$ $1216 \AA$ line should be found within the SALT-RSS spectral sensitivity ( $>3200 \AA$ ), and be used to model gamma-ray absorption from FSRQ above $25.6 \mathrm{GeV}$ (through the $\gamma \gamma \rightarrow e^{ \pm}$pair production process). White et al. [25] have confirmed eight objects from the VIDEO survey to be quasars, and obtained their spectra. One of these objects is a source at a redshift $z=2.7$, and is shown in Figure 5. At this redshift, the Ly $\alpha$ line is visible arund $4500 \AA$. 


\section{Summary}

In this paper, I briefly described possible studies that can be carried out by combining observations with Fermi-LAT and SAAO telescopes, and particularly using SALT-RSS for monitoring spectral evolutions of FSRQs during flaring episodes. Performing observations of FSRQs in such a multiwavelength context - that can also be extended to the radio, $\mathrm{X}$-ray and $\mathrm{TeV}$ domains would allow us to probe different physics processes related to AGN phenomenology, using independent and complementary observations.

\section{Acknowledgement}

I want to thank the organizers of the SSC 2015 conference for giving me the opportunity to present this work, and for their financial support to attend the conference.

\section{References}

[1] F. Acero, M. Ackermann, M. Ajello, et al., Fermi Large Area Telescope Third Source Catalog, ApJS, 218 (2015), 23 (41pp)

[2] M. Ackermann, M. Ajello, L. Baldini, et al., Fermi Gamma-ray Space Telescope Observations of Gamma-ray Outbursts from 3C 454.3 in 2009 December and 2010 April, ApJ, 721 (2010), pp. 1383-1396

[3] M. Ackermann, M. Ajello, W. B. Atwood et al., The Third Catalog of Active Galactic Nuclei Detected by the Fermi Large Area Telescope, ApJ, 810 (2015), 14 (34pp) ),

[4] M. Ackermann, M. Ajello, W. B. Atwood, et al., 2FHL: The Second Catalog of hard Fermi-LAT sources, ApJS, 222 (2016), 5 (19pp)

[5] A. A. Abdo, M. Ackermann, M. Ajello, et al., Fermi Gamma-ray Space Telescope Observations of the Gamma-ray Outburst from 3C454.3 in November 2010, ApJL, 733 (2011), L26 (7pp)

[6] W. B. Atwood, A. A. Abdo, M. Ackermann, et al., The Large Area Telescope on the Fermi Gamma-Ray Space Telescope Mission, ApJ, 697 (2009), pp. 1071-1102

[7] R. J. G. Britto, S. Razzaque, and B. Lott, on behalf of the Fermi-LAT Collaboration, Spectral Studies of Flaring FSRQs at GeV Energies Using Pass 8 Fermi-LAT Data, in Proceedings of the Fifth Fermi Symposium, arXiv:1502.07624 [astro-ph.HE] (2015), 7pp

[8] R. J. G. Britto, S. Buson, B. Lott, S. Razzaque, and E. Bottacini, Fermi-LAT Observations of 2014 May-July outburst from 3C 454.3, Submitted to ApJ, arXiv:1511.02280 [astro-ph.HE] (2015), 15pp

[9] D. A. H. Buckley, G. P. Swart, and J. G. Meiring, Completion and commissioning of the Southern African Large Telescope, in Proceedings of the SPIE, 6267 (2006), id. 62670Z, Ground-based and Airborne Telescopes, Edited by L. M. Stepp

[10] D. A. H. Buckley, J. B. Breytenbach, A. Kniazev, et al., SALT spectroscopy of the flaring blazar J141922.55-083832.0, The Astronomer's Telegram, 7167 (2015), 1

[11] E. B. Burgh, K. H. Nordsieck, H. A. Kobulnicky, et al., Prime Focus Imaging Spectrograph for the Southern African Large Telescope: optical design, in Proceedings of the SPIE, 4841 (2003), pp. 1463-1471, Instrument Design and Performance for Optical/Infrared Ground-based Telescopes, Edited by M. Iye, A. F. M. Moorwood 
[12] D. P. Cohen, R. W. Romani, A. W. Filippenko, et al., Temporal Correlations between Optical and Gamma-Ray Activity in Blazars, ApJ, 797 (2014), 137 (11pp)

[13] R. A. Edelson and J. H. Krolik, The discrete correlation function - A new method for analyzing unevenly sampled variability data, ApJ, 333 (1988), pp. 646-659

[14] J. D. Finke, Modeling Fermi Large Area Telescope and Multiwavelength Data from Blazars, Proceedings of Science, POS (HEASA2 015 ) 006 (2015), 21pp, arXiv:1602.05965 [astro-ph.HE], to appear in the proceedings of the 3rd Annual Conference on High Energy Astrophysics in Southern Africa (HEASA2015), 18-20 June 2015, Johannesburg, South Africa, Eds. M. Boettcher, D. Buckley, S. Colafrancesco, P. Meintjes and S. Razzaque

[15] L. Fuhrmann, S. Larsson, J. Chiang, et al., Detection of Significant cm to sub-mm Band Radio and $\gamma$-Ray Correlated Variability in Fermi Bright Blazars, MNRAS, 441 (2014), pp. 1899-1909

[16] M. Hayashida, K. Nalewajko, G. M. Madejski, et al., Rapid Variability of Blazar 3C 279 during Flaring States in 2013-2014 with Joint Fermi-LAT, NuSTAR, Swift, and Ground-Based Multiwavelength Observations, ApJ, 807 (2015), 79 (18pp)

[17] J. León-Tavares, V. Chavushyan, V. Patiño-Álvarez, et al., Flare-Like Variability of the Mg II $\lambda 2800$ Emission Line in the $\gamma$-Ray Blazar 3C 454.3, ApJL, 763 (2013), L36 (6pp)

[18] V. Lipunov, E. Gorbovskoy, V. Kornilov, et al., MASTER: very bright blazar flare, The Astronomer's Telegram, 7133 (2015), 1

[19] J. Poutanen and B. Stern, GeV Breaks in Blazars as a Result of Gamma-ray Absorption Within the Broad-line Region, ApJL, 717 (2010), pp. L118-L121

[20] S. Saito, Ł. Stawarz, Y. T. Tanaka, et al., Very Rapid High-amplitude Gamma-Ray Variability in Luminous Blazar PKS 1510-089 Studied with Fermi-LAT, ApJL, 766 (2013), L11 (6pp)

[21] B. Stern and J. Poutanen, The Mystery of Spectral Breaks: Lyman Continuum Absorption by Photon-Photon Pair Production in the Fermi GeV Spectra of Bright Blazars, ApJ, 794 (2014), 8 (7pp)

[22] Y. Tachibana, N. Kawai and S. Pike, on behalf of the MAXI Team and MITSuME Team, $A$ Correlation Between Optical, X-ray, and Gamma-ray Variations in Blazar 3C 454.3, in proceedings of the Fifth Fermi Symposium, arXiv:1502.03610 [astro-ph.HE] (2015), 6pp

[23] R. C. Telfer, W. Zheng, G. A. Kriss, and A. F. Davidsen, The Rest-Frame Extreme-Ultraviolet Spectral Properties of Quasi-stellar Objects, ApJ, 565 (2002), pp. 773-785

[24] C. M. Urry and P. Padovani, Unified Schemes for Radio-Loud Active Galactic Nuclei, PASP, 107 (1995), pp. 803-845

[25] S. V. White, M. J. Jarvis, B. Häußler, and N.Maddox, Radio-quiet quasars in the VIDEO survey: evidence for AGN-powered radio emission at $S_{1.4 G H z<1} m J y, M N R A S, 448$ (2015), pp. 2665-2686 